\title{
Conjunctival trauma following inadvertent use of nail glue containing cyanoacrylate, leads to patient education pamphlet
}

\author{
BY ETTY BITTON, OD, MSC, FAAO
}

\section{ABSTRACT \\ Similarities in packaging, bottle size and cap design of nail glue with ophthalmic products has led to patient confusion and inadvertent use of nail glue causing ocular trauma both in the adult and pediatric population. Despite efforts and pressures from several reports, little has been achieved with respect to unregulated health and beauty products. This case report describes a conjunctival trauma caused by inadvertent use of nail glue and highlights the importance of patient education. Basic precautions, such as reading the label of ophthalmic medication under proper lighting and with adequate correction coupled with the segregation of ophthalmic products from others in the medicine cabinet, could prevent ocular injury. To this end, an awareness pamphlet has been created to bring further awareness to this issue.}

Keywords: nail glue, ocular trauma, cyanoacrylate, patient education

\section{Introduction}

Tumerous drops are commer1 cially available for relief of ocular afflictions such as allergies, dry eye, contact lens related discomfort, glaucoma and ocular infections. Containers, similar in shape and size to eye drops are also available for other purposes, one being nail adhesive/ glue. Cyanoacrylate ${ }^{1}$, a synthetic adhesive found in nail glue, can cause strong adhesion of tissues with which it comes in contact, such as the eyelids or conjunctiva. Due to its inherent adhesive properties, cyanoacrylate has been reported to be useful in external ophthalmic surgical procedures such as sealing corneal perforations and tarsorraphy., ${ }^{2,3}$ Due to its rapid polymerization with water, a minimal amount is applied to a dried surface for tissue adhesion. ${ }^{1}$
Similarities in packaging of nail glue to ophthalmic products can lead to patient confusion and potentially harmful ocular trauma. The American Academy of Ophthalmology (AAO) initiated, in 1996, a colour coding system for the caps and labels of different new classes of ophthalmic solutions to minimize errors. ${ }^{4}$ Table 1 summarizes the colour coding system which is updated regularly as classes of drugs are introduced.

\section{TABLE 1}

Colour scheme system for ophthalmic solution caps

\begin{tabular}{|l|l|c|}
\hline Colour & Description & Pantone colour \\
\hline Tan & Anti-infectives (antibiotics, antivirals, antifungals) & 467 \\
\hline Pink & Anti-inflammatories or steroids & 197 \\
\hline Grey & Non-steroidal anti-inflammatories (NSAID) & 4 \\
\hline Red & Mydriatics/cycloplegics & 1797 \\
\hline Green & Miotics & 348 \\
\hline Yellow & Beta-blockers & Yellow C \\
\hline Blue & Beta-blocker combinations & 281 \\
\hline Purple & Adrenergic agonist & 2583 \\
\hline Orange & Carbonic anhydrase inhibitors & 1585 \\
\hline Turquoise & Prostaglandin analogues & 326 \\
\hline
\end{tabular}

Source: www.aao.org 


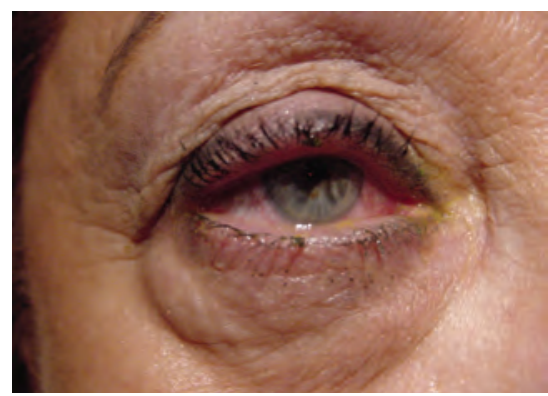

Figure 1: Lid swelling associated with inadvertent instillation of nail glue in the eye.

Similarities in size and shape of ophthalmic containers have lead to errors both by patients and medical personnel. Other health and beauty products, such as nail glue or superglue, with similar sizing to eye drops, have also been instilled inadvertently, both in adults and children. ${ }^{5-12}$ Thankfully, since cyanoacrylate is synthetic and nonbiodegradable, spontaneous rejection of the glue occurs with relatively favorable prognosis dependent on the tissue affected. ${ }^{13,14}$

Patient education can be the first line of defense in preventing potential harmful ocular trauma. Simple safeguards can be emphasized to patients, such as separation of all ophthalmic products from other health items as well as keeping all medications away from children. This case report highlights the preventative measures needed to avoid ocular injuries by inadvertent instillation of non-ocular health and beauty products, such as nail adhesive for artificial nails. As a result of this case report, a patient education pamphlet was designed to inform patients on how to prevent injuries in the home due to similarities in product appearance.

\section{Case report}

A 64 year old female was searching for eye drops to relieve some ocular discomfort after a late night out following removal of her soft contact lenses (CL). Confused by uncorrected vision, fatigue and similar looking containers in the dimly lit washroom, the patient inadvertently instilled nail adhesive in her right eye instead of the intended eye drops. The resulting severe burning and tearing provoked the patient to quickly blink and rinse her eye profusely. She reported that her lashes were stuck together for several minutes following the incident. The patient presented for consultation only

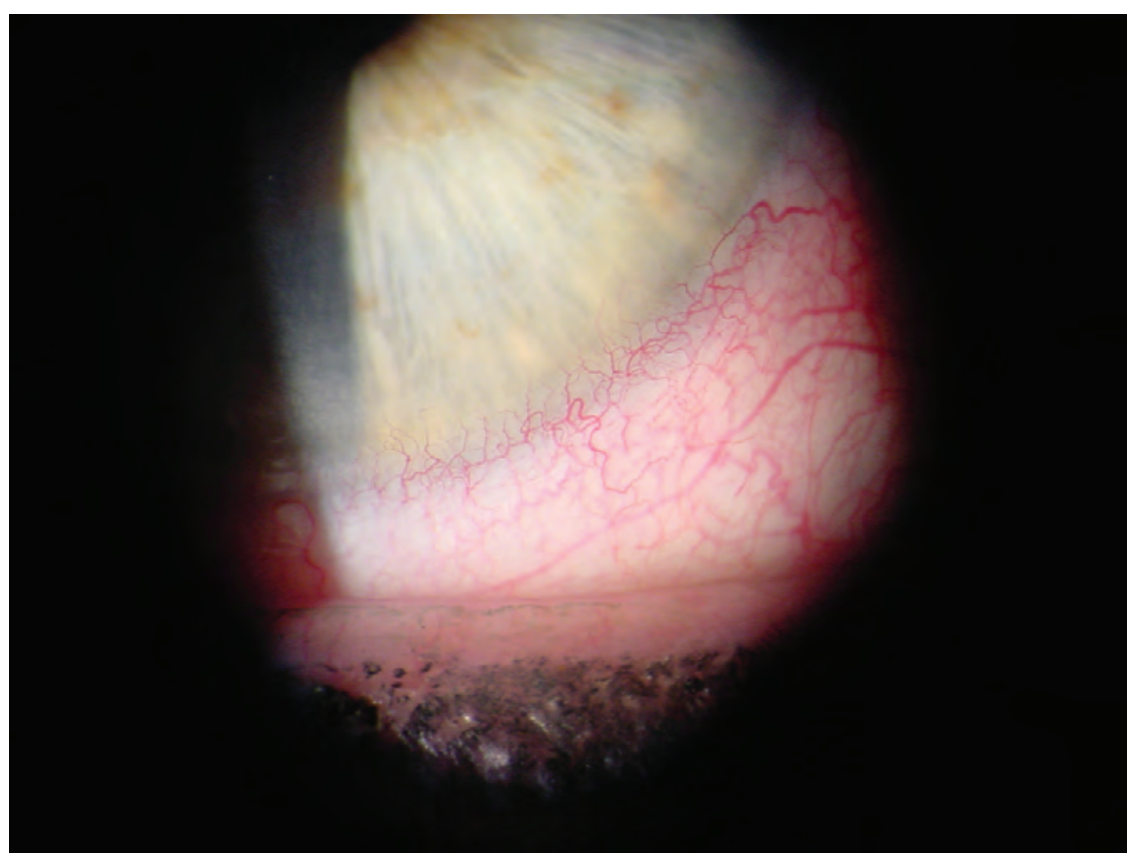

Figure 2: Limbal hyperemia associated with ocular irritation from nail glue instillation.
36 hours after the incident and reported progressive improvement of the condition, however the lids remained very swollen.

Upon presentation, the patient's symptoms included lid swelling of the right eye with mild ocular discomfort. The patient had ceased CL wear and reported no visual disruption. Presenting best corrected vision were good (OD $6 / 6^{-2}$, OS 6/6-1, OU 6/6). Slit lamp examination (SLE) of the right eye revealed a mild periorbital lid edema (Figure 1), a moderate (grade 2) bulbar conjunctival hyperemia (Figure 2) with a mild limbal congestion, a superior conjunctival hyperemia and a $6 \mathrm{~mm}$ wide tissue trauma, grade 4 (zone $1,2,3$ and 4 as defined using the CCLRU grading scales) ${ }^{15}$ (Figure 3). No foreign body was found under the upper lid. The lashes, palpebral margin, cornea and anterior 


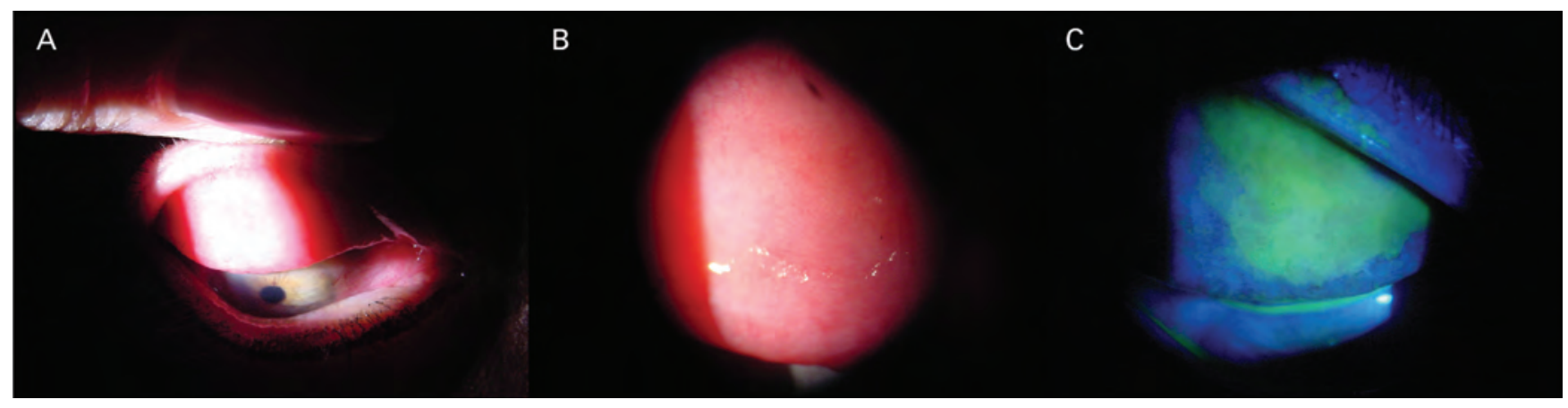

Figure 3: Conjunctival trauma related to unintentional use of nail glue in the eye. The superficial layers were sloughed off resulting in a large area (6mm wide) of fluorescein uptake by zones 1-3 of the palpebral conjunctiva.

chamber of the right eye revealed no anomalies or staining with fluorescein. The left eye revealed no anomalies. A mild meibomian gland (MG) obstruction was noted in both eyes, but the record revealed that this was a pre-existing condition. The remainder of the ocular exam was unremarkable.

The clinical management included an ocular lavage of the upper tarsus with a rinsing solution (Multipurpose Sensitive Eyes ${ }^{\mathrm{TM}}$, $\mathrm{B}+\mathrm{L}$ ) during the consultation and subsequent use of unpreserved ocular lubricants (BIONTM Tears, ALCON) qid for 48 hrs. The patient was instructed not to use make-up or contact lenses until the next follow-up visit.

At the 48 hour follow-up visit, (now four days post-trauma), symptoms were vastly improved. No stinging or burning was reported and the patient reported that her affected eye "felt and looked" better. Best corrected acuities were OD/OS 6/6 $6^{-1}$, OU 6/6. The SLE of the right eye revealed minimal lid edema, slight bulbar conjunctival

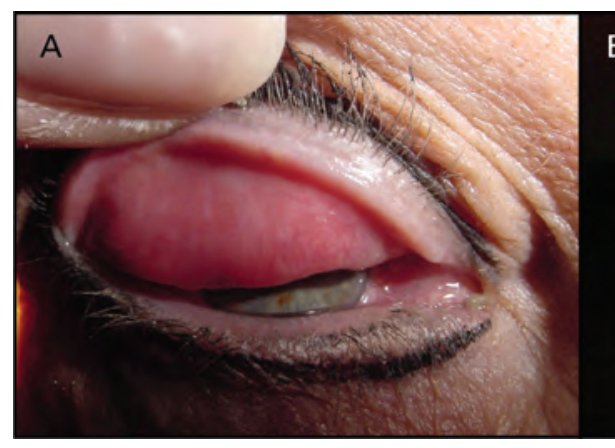

B

Figure 4: Regressing conjunctival trauma at the 48-hour follow up visit (4 days post trauma).

hyperemia. Figure 4 reveals the slowly regressing superior conjunctival trauma (6 $\mathrm{mm}$ wide), grade 3 (zone 2, 3). The lashes, palpebral margin, cornea, anterior chamber revealed no anomalies. Clinical management included continuation of unpreserved tear lubricants qid for 7 days, no CL wear and a follow-up in one week.

The patient was lost to followup due to hip replacement surgery followed by bed rest and physiotherapy sessions. She consulted four months later with vast improvement of her ocular condition. The patient had reinitiated CL wear several weeks prior and had no associated symptoms. She also reported that she had separated her ophthalmic products from other products in her medicine cabinet. Her vision remained stable (OD 6/6, OS 6/6-1, OU 6/6) and a SLE revealed no anomalies in either eye (Figure 5) except for the mild preexisting MG obstruction. Clinical management included lubricating drops, (Systane ${ }^{\mathrm{TM}}$, ALCON) prn, to aid with tear film stability, which was a bit worse in the right eye (tear break up timeTBUT OD 2-3 sec, OS $5 \mathrm{sec}$ ) and warm compresses and gland expression for the mild MG dysfunction. Routine follow ups at 


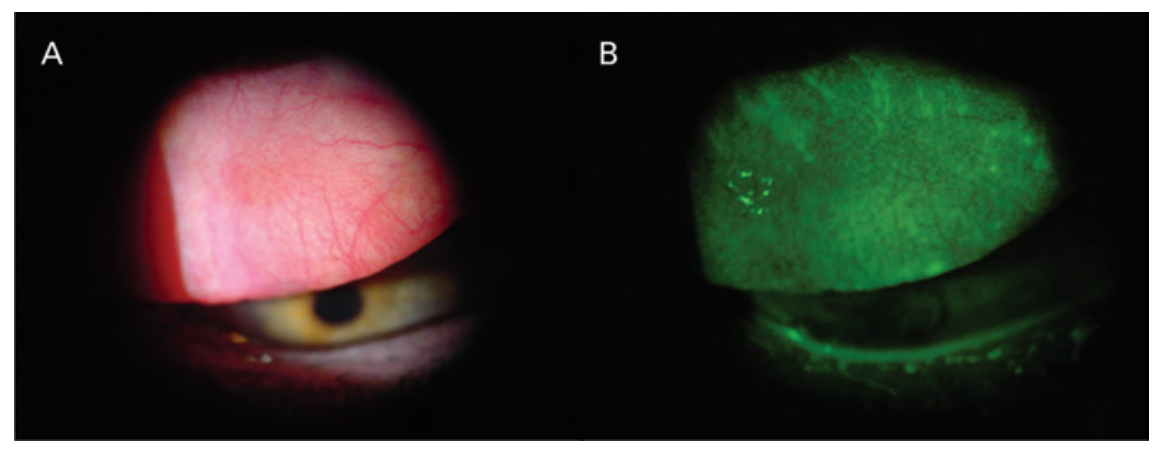

Figure 5: Resolved conjunctival trauma 4 months post trauma.

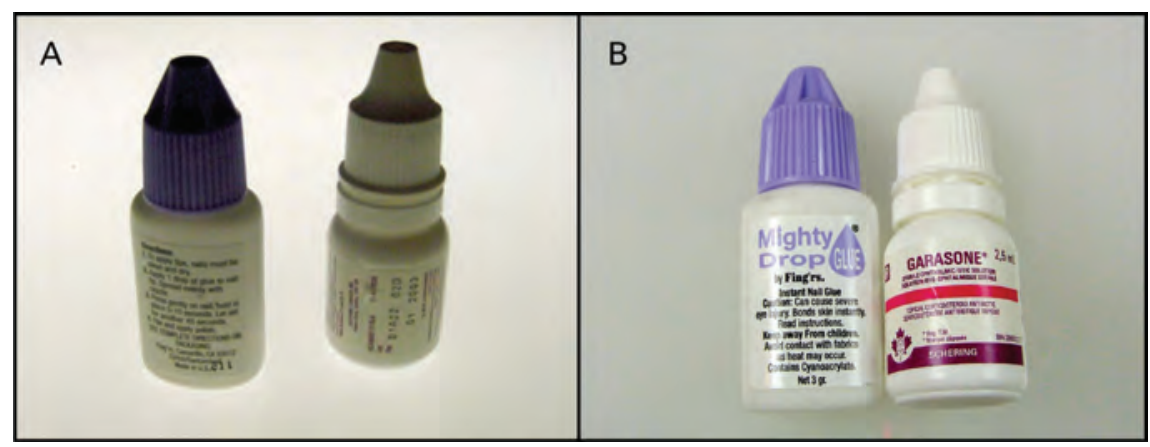

Figure 6: Similarities of bottle size and caps between nail glue and an ophthalmic solution in (A) poor lighting conditions and (B) normal conditions.

annual visits were recommended.

The patient was asked to bring the eye drop and the nail adhesive containers for comparison. Figure 6 shows the similarities in bottle size, shape and bell-shaped bottle cap, which were contributory factors, adding to the confusion. Although the caps were of different colours, the uncorrected vision following CL removal, dim lighting of the room and the tired state of the patient compounded by the fact that the containers were side by side were all contributory to the confusion. Further evaluation of the eye drops revealed that in fact they were not intended for CL related relief, but were a topical anti-infective (antibiotic steroid) that was used for a previous eye infection and were past their expiry date. This revelation prompted a discussion with the patient about proper identification of ophthalmic products and appropriate placement of products in the medicine cabinet and the disposal of expired medication.

\section{Discussion}

The similarities in packaging of ophthalmic preparations and other beauty products, such as nail adhesive, have been the cause of numerous unnecessary ocular trauma, reported both in the adult and pediatric population..$^{5-11}$ Despite efforts to bring awareness to this issue, similarities in product
A

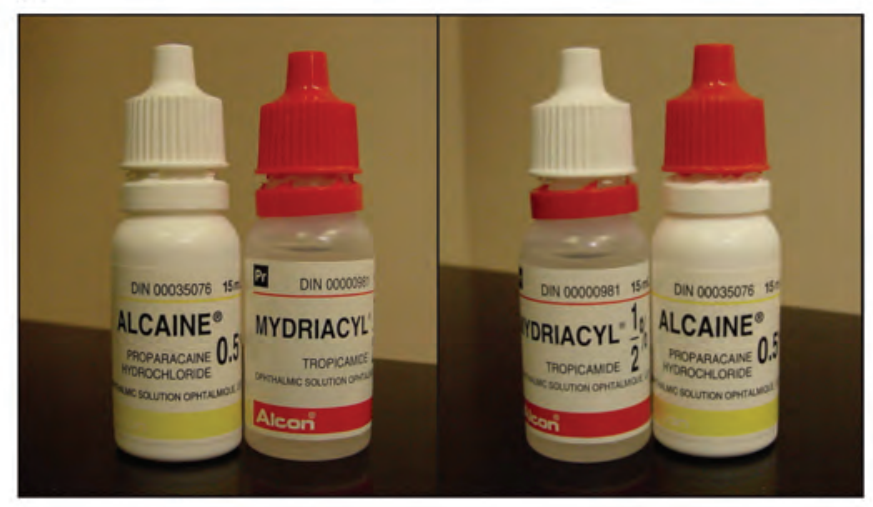

\section{B}

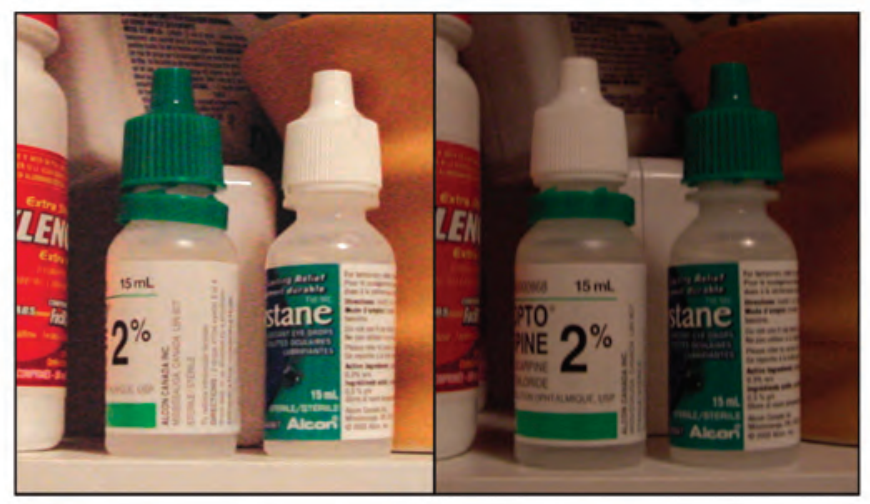

Figure 7: Interchangeable caps and similar colouring can cause confusion between drops intended for (A) pupillary dilation and topical anesthetics or between (B) ocular lubricants and glaucoma medication. 


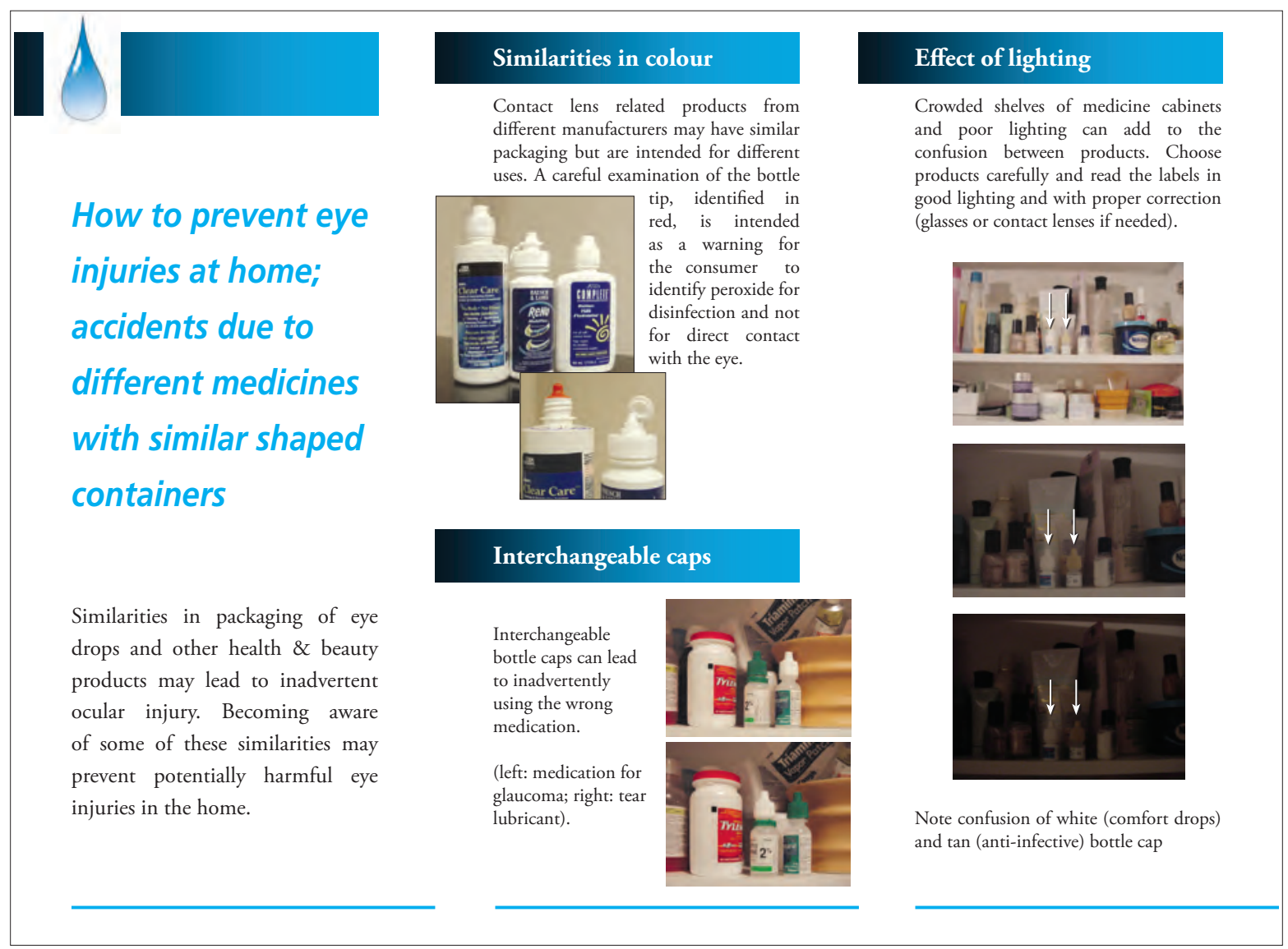

Patient Education Pamphlet

packaging continues to threaten patients' safety.

Although colour coding the bottle caps has improved the identification of ophthalmic products both for in-office use and home purposes, interchangeable caps can still lead to mistakes. For example, attention in the practitioner's office in recapping bottles intended for pupillary dilation is extremely important so as not to inadvertently dilate a patient at risk for angle closure. At home, interchangeable caps may lead to instillation of tear lubricants or anti-infectives instead of glaucoma medication. Figure 7 demonstrates interchangeable caps between drops intended for papil- lary dilation and topical anesthetics and between ocular lubricants and glaucoma medication. In the latter case, the green cap looks well coupled with the ocular lubricant since the label has a similar colour.

Similarities in package appearance amongst leading contact lens solutions can also lead to inadvertent instillation of the wrong solution, such as hydrogen peroxide instead of multipurpose solutions. Manufacturers have attempted to address the problem by making the bottle tip red, instead of the cap, to identify the hydrogen peroxide solution. Red is usually interpreted by patients as an alert to potential harm and can momentarily stop/ alert the patient to properly read the label. This induced moment of hesitation can prevent unnecessary hydrogen peroxide burns of the corneal and conjunctival surfaces.

Suggestions for improved packaging have included different size and shape containers, non-interchangeable caps, colour coding caps and/or containers, child safety caps and Braille warning on bottles ${ }^{5-11,16,17}$. A different bottle cap design for nail adhesive has been suggested incorporating a child safety feature such as alignment of arrows which would require good lighting and acuity prior to opening. ${ }^{6}$ In addition to changes in packaging and bottle cap design, 
Five simple precautions !

1. Use good lighting and proper correction when selecting medications.

2. Always read the label to make sure you have the correct product.

3. Beware of similarities in packaging, bottle size, shape and interchangeable caps.

4. Separate your eye medication from all other products.

5. Always keep medication, including health and beauty products, such as nail glue, away from children.

If you have any questions concerning any eye care products, check with your eye care practitioner.

Your eye care practitioner is:

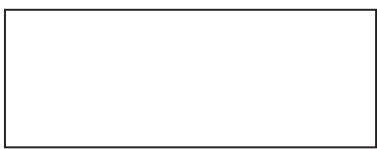

Created by Etty Bitton, OD, MSc, FAAO Associate professor
École d'optométrie, Université de Montréal Graphic design: Denis Latendress
Separate similar colour caps

Although industry standards exist for colour coding of bottle caps for eye medications, this is not the case for other health $\&$ beauty products, such as nail glue. Separating eye medications will prevent inadvertent use of nail glue or other products (both in adults and curious children).
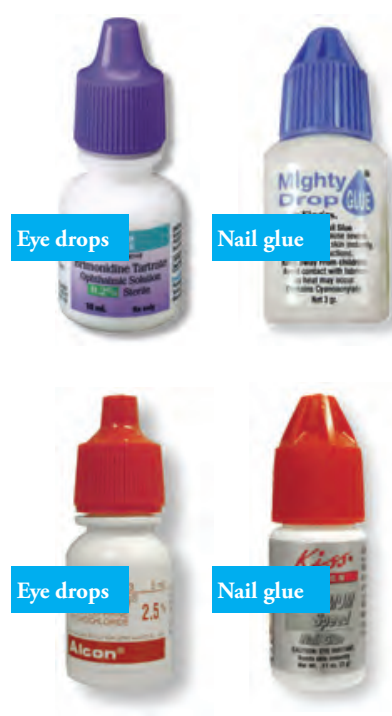

Organize your cabinet

An ounce of prevention goes a long way. Organize your medicine cabinet and separate the products intended for the eyes from all other products.

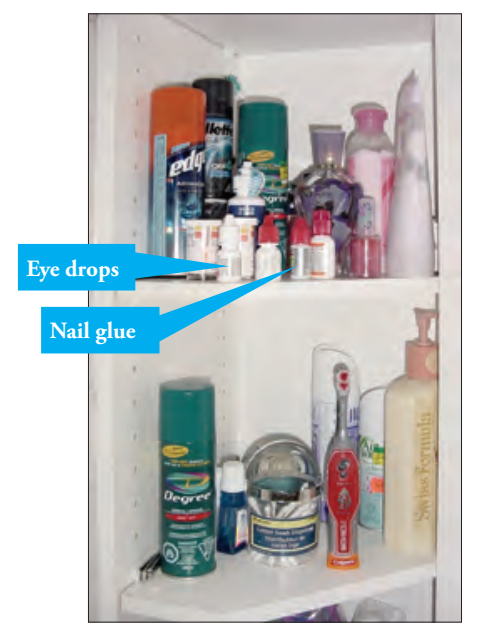

patient awareness must be included in the overall prevention of these types of ocular trauma.

Patient education should include the segregation of all ophthalmic preparations and CL solutions from other health and beauty products or other household items. Precaution such as carefully reading the contents of the container and its intended use in a well lit environment with the best correction possible could prevent injury. Proper patient instruction in respecting the coloured bottle cap is important in the proper management of the patient's ocular condition, especially in the presence of more than one ophthalmic medica- tion. Furthermore, all medication, prescribed or over-the-counter, should be kept out of the reach of children.

In an attempt to bring further awareness to the errors caused by similarities in packaging and labeling of ophthalmic medications, some organizations have asked for people to sign an e-petition on the "look alike, sound alike" (LASA) issue. ${ }^{18}$ Hopefully this will bring further attention to this issue that will ultimately benefit patients' safety in the use of ophthalmic products. A collaborative effort including manufacturers, health organizations, healthcare providers and patients should address packaging issues (colour, font size for the visually impaired, similarities with other products), safety features (caps, expiry dates) and establish a quality assurance system to assess the effectiveness of these new efforts.

To assist the eye care community in bringing further awareness to this issue, a patient awareness pamphlet has been created entitled "Preventing eye injuries in the home: Avoiding errors due to similarities in appearance". The pamphlet illustrates packaging similarities, interchangeable bottle caps, similarly coloured caps with other health and beauty products and the importance of organizing 
the medicine cabinet to segregate ophthalmic products. The pamphlet highlights the importance of lighting when reaching for any kind of medication so as to properly identify the intended container. Eye care practitioners can make the pamphlets readily available to their patients and staff both in the office as a hard copy or electronically via electronic newsletters or as a link on their websites.

Clinic environments such as hospitals, optometry school clinics and other multidisciplinary clinics have numerous people interacting with each other. Bottles of diagnostic medications are usually left in examining rooms and vigilance amongst workers in replacing the appropriate bottle caps and applying the necessary precaution when instilling eye drops of any kind is important to maintain patient safety. Interchangeable bottle caps render the discrimination of ophthalmic solutions by colour unreliable. There is no better universal precaution than accurately reading the label under proper illumination and with an adequate correction prior to instillation on the ocular surface.

\section{Summary}

Ocular injuries with inadvertent use of nail glue due to similarities in packaging, bottle size and cap design have been reported repeatedly in the literature and remain a concern for patient safety. This case report describes a conjunctival trauma caused by inadvertent use of nail glue and highlights the importance of patient education in preventing ocular trauma by segregating ophthalmic preparations. Basic precautions such as reading the label of ophthalmic medication under proper lighting and with adequate correction coupled with the segregation of ophthalmic products from others in the medicine cabinet could have prevented the injury. To that end, an awareness pamphlet has been created to bring further awareness to this issue.

\section{References}

1. Chan SM, Boisjoly H. Advances in the use of adhesives in ophthalmology. Curr Opin Ophthalmol 2004;15:305-10.

2. Sharma A, Kaur R, Kumar S, al. e. Fibrin glue versus n-butyl-2cyanoacrylate in corneal perforations. Ophthalomology 2003;110:291-9.

3. Ehrenhaus M, P. DA. Improved technique for temporary tarsorraphy with a new cyanoacrylate gel. Arch Ophthalmol 2003;121:1336-7.

4. AAO, American Academy of Ophthalmology. Color codes for topical ocular medications. Available from: http:/ /www.aao.org. Accessed on July 6, 2010 .

5. Needham A, Natha S, Kaye S. Similarities in the packaging of cyanoacrylate nail glue and ophthalmic preparations: an ongoing problem. BJO 2001;85:496-7.

6. Good A, McCabe S. Superglue accidents and the eye-causes and prevention. BJO 1994;78:802.

7. Knight I. Mistaken eye drops and subsequent instillation of superglue. Eye 2001;15:663.

8. Fraunfelder F. Drug-packaging standards for eye drop medication. Arch Ophthalmol 1988;106:1029.

9. Silverman C. Corneal abrasion from accidental instillation of cyanoacrylate into the eye. Arch Ophthalmol 1988;106:1029-30.

10. Raynor L. Treatment of inadvertent cyanoacrylate tarsorrhaphy. Arch Ophthalmol 1988;106:1033.

11. Ling R, Villalobos R, Latina M. Inadvertent instillation of hemoccult developer in the eye. Arch Ophthalmol 1988;106:1033-4.

12. McLean C. Ocular superglue injury. J Accid Emerg Med 1997;14(3):40-1.

13. Rohrbach J, Wohlrab T, Nolle B, Schlote T. Cyanoacrylate injuries in the eye. Ophthalomologe 2000;97(12):87880.

14. Rohrbach J, Schlote T, Wohlrab T. Eye injury caused by superglue. Klin Monatsbl Augenheilkd 2000;216(1): 57-8.

15. Terry R, Schnider C, Holden B, et al. CCLRU standards for success of daily and extended wear contact lenses. OVS 1993;70:234-43.

16. Desai S, Teggihalli B, Bhola R. Superglue mistaken for eye drops. Arch Dis Child 2005;90:1193.

17. Spencer T, Clark B. Selfinflicted superglue injuries. MJA 2004;181(6):341.

18. AAEECE, The American Association of Eye and Ear Centers of Excellence. LASA e-petition. Available from: http:/ / www.nyee.edu/lasa-e-petition. html. Accessed on July 10, 2010.

\section{Acknowledgements}

I would like to thank Mr. Denis

Latendresse for the design of the

Awareness Pamphlet

Etty Bitton

Associate professor

École d'optométrie,

Université de Montréal

email: etty.bitton@umontreal.ca

The pamphlet is downloadable

by contacting the author. 